\title{
Mechanical cardio-pulmonary support in critical care; early experience with Extracorporeal Membrane Oxygenation (ECMO) in Sri Lanka
}

\author{
Harischandra DVT, Withanaarachchi K, Kumara MT, Firmin RK \\ Teaching Hospital, Karaptiya, Galle, Sri Lanka.
}

Correspondence: Dr. Tolusha Harischandra

e-mail: tolushah@gmail.com

\begin{abstract}
Introduction
Extra corporeal membrane oxygenation (ECMO) is a form of temporary support of the lungs and/or heart for severe respiratory and/or cardiac failure not amenable to conventional forms of treatment. It evolved as an extension of the technology involved in cardiopulmonary bypass in heart surgery but in this case for long term use in the intensive care setting.
\end{abstract}

Here we present the first successful use of ECMO reported in Sri Lanka.

\section{Case Report}

A $3.5 \mathrm{~kg}$ baby boy was born via forceps delivery at 39 weeks of gestation. Thick meconium was noted at birth. His Apgar score was 4 at 1 minute and after resuscitation 9 at 5 minutes. His respiratory distress gradually worsened and he was transferred to the neonatal intensive care unit (NICU) at 6 hours of age.

The baby was intubated and ventilated and administered surfactant. As he remained acidotic with high $\mathrm{pCO}_{2}$ and low $\mathrm{pO}_{2}$ levels despite conventional ventilation High Frequency Oscillatory Ventilation (HFOV) was initiated. Satisfactory blood pressure control was achieved with minimal inotropes. Echocardiography showed severe pulmonary hypertension with a patent ductus arteriosus (PDA) and a small atrial septal defect (ASD), both with bidirectional shunting. A diagnosis of meconium aspiration syndrome (MAS) and persistent pulmonary hypertension of the newborn (PPHN) was made.

At day one, the ECMO team was consulted as the oxygenation index (OI) was 54. However, with
HFOV, magnesium sulphate infusion, sildenafil and inotropes, the OI improved to 21 , and it was decided to continue conventional treatment. As the Creactive proteins and White cell count were rising, intravenous gentamicin and cefotaxime were started to treat secondary infections.

On the day 5, although the OI was 28 , the ventilator settings remained high and the blood gases were unsatisfactory. Echocardiography revealed a pulmonary pressure gradient of $70 \mathrm{mmHg}$ while the mean arterial pressure was $60 \mathrm{mmHg}$ with a bidirectional shunting across the ASD. The PDA had closed. Computerized tomography scan of brain and coagulation screening were normal. At this point, a second consultation was made with the ECMO team and a decision was made to institute ECMO.

The Levitronix Centri Mag ECMO machine was set up and the circuit was primed in the NICU by the perfusionists. The baby was sedated and paralyzed. Venoarterial ECMO was instituted by the cardiothoracic surgical team at the bedside via an open method, cannulating the right common carotid artery and right internal jugular vein using two $10 \mathrm{~F}$ Medtronic cannulae (Figure 1). The ventilator settings were adjusted to rest settings on conventional SIMV mode.

The vital signs, blood gases, coagulation tests including Activated Clotting Time (ACT), ECMO flow and gas sweep were monitored and appropriate changes were made to maintain normal parameters. Daily chest radiographs showed gradual improvement. No organisms were found on the septic screen. Echocardiography showed a steady reduction of pulmonary pressures to $28 \mathrm{mmHg}$ by the age of 8 days. The shunt became left to right across the small ASD. 


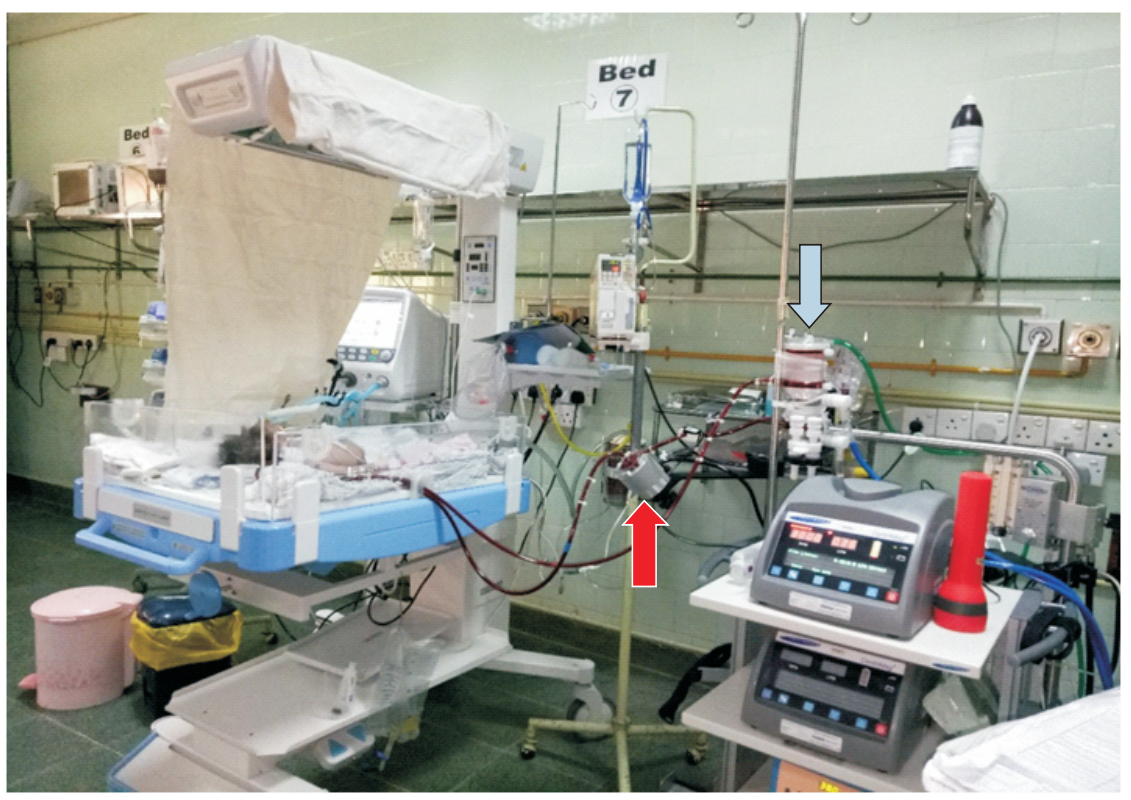

Figure 1: Neonate on ECMO: The centrifugal pump (the artificial heart-red arrowhead) sends deoxygenated blood through an oxygenator (the artificial lung- blue arrowhead) where it is oxygenated and returned to the body.

Fifty seven hours after initiating ECMO, a coagulopathy was noted with oozing from the neck cannulation site. As the respiratory and cardiac functions had recovered by then, the baby was weaned off ECMO. The trialing-off ECMO using a retrograde flow method was successful, and therefore, decannulation was done under sterile conditions by the cardiothoracic surgical team. The total ECMO run was 78 hours.

Forty hours later, the baby was weaned off ventilation and extubated. At 12 days of age the mother was able to initiate breast feeding and at 28 days, was discharged from hospital.

\section{Discussion}

In 1974, Dr. Robert Bartlett's performed the first ECMO on a critically ill neonate with meconium aspiration. She was later named Esperanza (meaning "hope" in Spanish) by the nurses and is alive today (1). Since then 86,287 patients have undergone ECMO around the world, of which the largest group consists of 29,942 neonatal respiratory patients (2).

The hypoxia and acidaemia of meconium aspiration constrict the pulmonary blood vessels, reduces the pulmonary blood flow, produces a right-to-left shunt and worsens hypoxia. ECMO effectively improves oxygenation and breaks this vicious cycle that perpetuates PPHN, as demonstrated here.

Our patient illustrates the philosophy on which ECMO has evolved: i.e., to support the heart and lungs to allow time or therapeutic intervention to allow for healing. During this time, ECMO prevents not only death from respiratory/cardiac failure, but also hypoxic brain damage and its dreaded sequelae: cerebral palsy.

This is our first success in ECMO, and marks a milestone in the history of cardiothoracic surgery, neonatology, pediatrics and critical care in Sri Lanka.

\section{Acknowledgements}

The authors thank Chalice Medical Ltd, UK for the loan of the ECMO machine, the Ministry of Health, Sri Lanka for the consumables and the multidisciplinary staff at Teaching Hospital Karapitiya for the care of the baby.

\section{References}

1. Bartlett RH. Esperanza. Presidential address. Trans Am Soc ArtifIntern Organs 1985; 31: 723-6.

2. Extracorporeal life support organization registry report. International Summary; January 2017. Available at: https://www.elso.org. 


\title{
Massive pericardial effusion in a patient during the recovery phase of dengue haemorrhagic fever
}

\author{
Palangasinghe DR, Samaranayake MD, Weerarathna TP \\ Professorial Medical Unit, Teaching Hospital Karapitiya, Galle, Sri Lanka.
}

Correspondence: Dr. Dhammika Palangasinghe

e-mail:dhammika27@yahoo.com

\section{Introduction}

Dengue haemorrhagic fever (DHF) is characterized by plasma leakage into peritoneal and pleural spaces. However few clinically significant pericardial effusions were reported in DHF $(1,2)$. Herein we report pericardial effusion with cardiac tamponade during the DHF recovery phase.

\section{Case Report}

A 30-year old male presented with fever, arthralgia, myalgia and headache for 4 days. On admission he was febrile (100.4 F).His pulse was $72 / \mathrm{min}$ with blood pressure (BP) of $120 / 80 \mathrm{mmHg}$. His heart sounds were audible in normal intensity with no murmurs. Rest of the clinical examination was unremarkable. Investigations on the $4^{\text {th }}$ day showed haemoglobin $14.6 \mathrm{~g} / \mathrm{dL}$, haematocrit 45 , platelet count $65,000 / \mathrm{uL}$ and white cell count (WBC) $4,200 / \mathrm{uL}$. On the $5^{\text {th }}$ day ultrasound scan revealed free fluid in the Morrison's pouch for which appropriate fluid therapy was instituted. The lowest platelet count $(12,000 / \mathrm{uL})$ and the highest haematocrit $(58 \%)$ were recorded on the $7^{\text {th }}$ day. Repeat ultrasound scan showed ascites, pleural effusions but no pericardial effusion. He was discharged on the following day with no fever, normal appetite, haematocrit and rising platelets. Five days after discharge he developed fever, dry cough, pleuritic type of chest pain, progressive dyspnea and was admitted to hospital next day.

He was febrile $\left(101{ }^{0} \mathrm{~F}\right)$, on readmission with regular pulse at a rate of $108 / \mathrm{min}, \mathrm{BP}$ was $110 / 70 \mathrm{mmHg}$ and jugular venous pulse (JVP) was elevated. He had soft heart sounds with no murmurs. Left lung base was dull to percussion with absent breath sounds. Electrocardiogram revealed diffuse ST elevations with low voltage complexes. Chest radiograph revealed cardiomegaly with well demarcated cardiac borders without radiographic evidence of pulmonary oedema or pleural effusions. Transthoracic echocardiogram revealed a moderate pericardial effusion. Other investigations revealed a platelet count of 312,000/uL, haemoglobin - $13.2 \mathrm{~g} / \mathrm{dL}$, WBC of $10,000 / \mathrm{uL}$ with neutrophil of $83 \%$. Dengue antibodies (IgM and $\operatorname{IgG}$ ) were positive. C reactive protein was $96 \mathrm{ng} / \mathrm{dL}$ and ESR was $90 \mathrm{~mm}$ in $1^{\text {st }}$ hour. He was commenced on intravenous ceftriaxone $1 \mathrm{~g}$ bd and clarithromycin $500 \mathrm{mg}$ bd. On the following day he developed increasing dyspnea, tachycardia, elevated JVP and hypotension suggestive of cardiac tamponade. Chest radiograph (Figure 1) and repeat echocardiogram showed a massive pericardial effusion. He underwent pericardiocentesis where $300 \mathrm{~mL}$ of straw colour fluid was removed. Pericardial fluid was an exudate with no atypical cells, negative gram stain, bacterial culture and acid fast bacilli. Adenosine deaminase level was normal. He was commenced on ibuprofen $400 \mathrm{mg}$ tid and prednisolone $30 \mathrm{mg}$ daily for a presumed Dressler's syndrome (DS). Antibiotics were omitted after 3 days once blood, urine and pericardial fluid cultures became negative. Echocardiogram performed 7 days after pericardiocentesis showed mild pericardial effusion. He was discharged on $10^{\text {th }}$ day after readmission (day 24 of illness)when chest radiograph (Figure 2) and echocardiogram revealed resolution of the pericardial effusion. His inflammatory markers improved and were normal at two weeks after discharge. Prednisolone and ibuprofen were tailed off over next 2 weeks as the repeat echocardiogram showed full resolution of the pericardial effusion. One month later he was apparently well and echocardiographic examination did not reveal recurrence of pericardial effusion. 


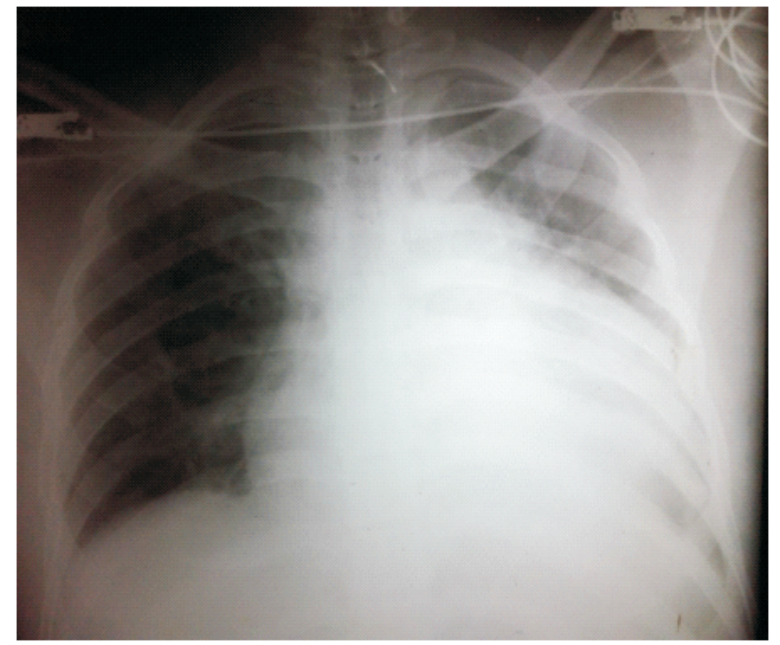

Figure 1: Chest radiograph on day 15 of the illness showing evidence of a massive pericardial effusion

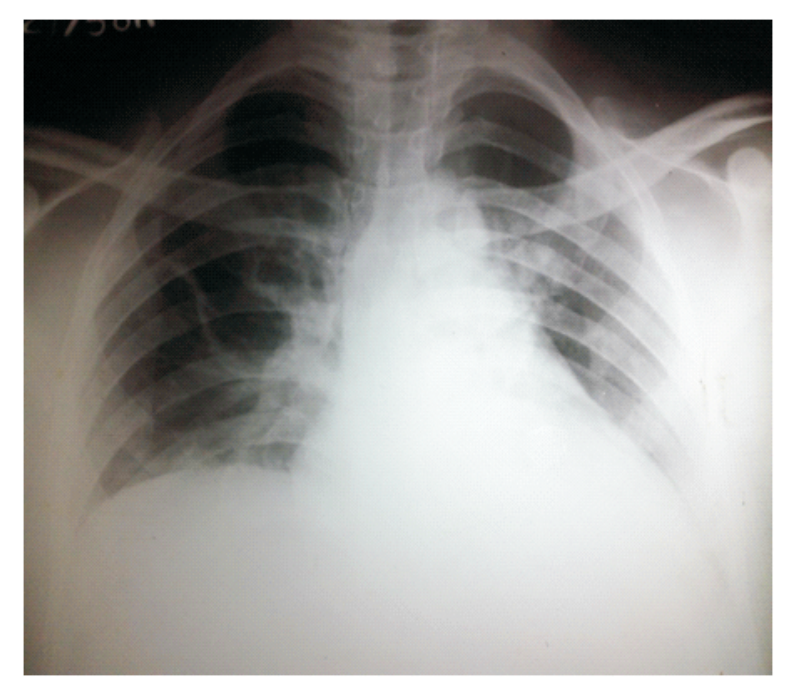

Figure 2: Chest radiograph after 10 days of treatment (day 25 of illness) showing resolution of pericardial effusion

\section{Discussion}

There were several cases of clinically significant pericardial effusions occurring during the critical phase of DHF $(1,2)$. The current case describes a pericardial effusion leading to cardiac tamponade in DHF during the recovery phase.

This patient who was discharged after recovering from DHF was readmitted with fever and progressive dyspnea. He had no evidence of hospital acquired bacterial infections (urosepsis, thrombophlebitis and endocarditis) which are known to occur in the recovery phase of dengue fever $(3,4)$.

He was commenced on antibiotics on the day 1 of second admission for suspected pyogenic pericarditis with effusion. Antibiotics were stopped 3 days later after ruling out a bacterial infection (negative blood culture, pericardial fluid gram stain and culture). He responded to steroids and NSAIDs with resolution of fever, chest pain and improvement of inflammatory markers. Pericardial fluid aspirate was an exudate with no malignant cells, negative gram stain, bacterial culture, normal ADA levels and negative culture for tuberculosis. Antinuclear antibody was negative making systemic lupus erythematosus unlikely which could have been precipitated with dengue (5). With the exclusion of other possibilities and response to anti-inflammatory therapy Dressler's syndrome was the most likely cause of pericardial effusion.

DS is an inflammatory pericarditis with or without pericardial effusion that occur secondary to injury (in the form of myocardial infarction, surgery, trauma or viral infections) to myocardium or pericardium $(6,7)$. The exact aetiology of DS remains unclear while autoimmune responses and viral infections have been considered as causes $(7,8)$.

Our case highlights the importance of considering DS as a differential diagnosis for pericardial effusion in a patient presenting with fever and dyspnea during the recovery phase of DHF.

\section{Conclusion}

Clinically significant pericardial effusions could occur with DHF not only during the critical phase but also during the recovery phase. High degree of suspicion is required for early detection and prompt treatment is necessary to reduce mortality.

\section{References}

1. Pelupessy JM, Allo ER, Jota S. Pericardial effusion in dengue haemorrhagic fever. Paediatr Indones. 1989 MarApr; 29(3-4): 72-5. 
2. Sophie Yacoub, Heiman Wertheim, Cameron P. Simmons, GavinScreaton\& Bridget Wills Cardiovascular manifestations of the emerging dengue pandemic. Nature Reviews Cardiology 20914; 11: 335-45 (doi:10.1038/nrcardio.2014.40)

3. R Premaratna, D Dissanayake, FHDS Silva, M Dassanayake, HJ de Silva. Secondary bacteraemia in adult patients with prolonged dengue fever. Ceylon Medical Journal 2015; 60: 10-12.

4. Kay C. See, Jason Phua, Hwee S. Yip, Leong L. Yeo, and Tow K. Lim. Identification of Concurrent Bacterial Infection in Adult Patients with Dengue. Am J Trop Med Hyg 2013 Oct 9; 89(4): 804-10.
5. SH Talib, SR Bhattu, R Bhattu, SG Deshpande, and DB Dahiphale Dengue fever triggering systemic lupus erythematosus and lupus nephritis: a case report. Int Med Case Rep J2013; 6: 71-5.

6. Burch, GE and Colcolough, HL. Postcardiotomy and post-infarction syndromes: A theory. Am Heart J 1970; 80: 290-1.

7. McCabe, JC, Ebert, PA, Engle, MA et al. Circulating heart-reactive antibodies in the post pericardiotomy syndrome. J Surg Res 1973; 14: 158-64.

8. Engle MA, Zabriskie JB, Senterfit LB, et al. Viral illness and the postpericardiotomy syndrome. A prospective study in children. Circulation 1980; 62: 1151. 